\title{
INDEX TO VOLUME 70
}

\section{RESEARCH ANNOUNCEMENTS}

Aanderaa, Stål. See Dreben, Burton.

Accola, R. Invariant domains for Kleinian groups, 412.

Alling, N. L. A proof of the corona conjecture for finite open Riemann surfaces, 110.

Amemiya, Ichiro. See Itô, Takashi.

Anderson, P. G. Cobordism classes of squares of orientable manifolds, 818 .

Arkowitz, M. and Curjel, C. R. The group of homotopy equivalences of a space, 293.

Babbitt, Donald. The Wiener integral and perturbation theory of the Schrödinger operator, 254.

Baily, W. L. and Borel, A. On the compactification of arithmetically defined quotients of bounded symmetric domains, 588.

Bajsanski, B. and Bojanić, R. A note on approximation by Bernstein polynomials, 675 .

B arratt, M. G. and Mahowald, M. E. The metastable homotopy of $O(n), 758$.

Barros-Neto, J. The Dirichlet problem for homogeneous elliptic operators in a half space, 798

Bass, Hyman. The stable structure of quite general linear groups, 429 .

Bass, H., Lazard, M. and Serre, J.-P. Sous-groupes d'indice fini dans $S L(n, Z), 385$

Baumslag, Gilbert and Steinberg, Arthur. Residual nilpotence and relations in free groups, 283.

Baxter, Glen and Hirschman, I. I., Jr. An explicit inversion formula for finite-section Wiener-Hopf operators, 820.

Beals, Richard. Nonlocal elliptic boundary value problems, 693.

Beals, R. W. A note on the adjoint of a perturbed operator, 314.

Bear, H. S. A strict maximum theorem for one-part function spaces and algebras, 642.

Beineke, L. W. and Harary, Frank. On the thickness of the complete graph, 618.

Berens, Hubert and Butzer, P. L. On the best approximation for singular integrals by Laplace-transform methods, 180.

Approximation theorems for semi-group operators in intermediate spaces, 689.

Bers, Lipman and Ehrenpreis, Leon. Holomorphic convexity of Teichmüller spaces, 761.

Bishop, Errett. Representing measures for points in a uniform algebra, 121.

Blakley, G. R. Homogeneous nonnegative symmetric quadratic transformations, 712 .

Bojanić, R. See Bajsanski, B.

Borel, A. See Baily, W. L.

de Branges, Louis and Rovnyak, James. The existence of invariant subspaces, 718.

Browder, F. E. Nonlinear elliptic problems. II, 299.

Continuity properties of monotone nonlinear operators in Banach spaces, 551.

Brown, Arlen and Pearcy, Carl. Structure theorem for commutators of operators, 779.

Brumer, Armand. Addendum to "Structure of hereditary orders," 185.

Butzer, P. L. See Berens, Hubert.

Carlitz, L. A problem in partitions related to the Stirling numbers, 275.

Chacon, R. V. Ordinary means imply recurrent means, 796.

Chakerian, G. D. and Stein, S. K. On the symmetry of convex bodies, 594.

Chang, C. C. Some nerw results in definability, 808.

Chen, Y. W. On the solutions of the wave equation in a quadrant of $R^{4}, 172$.

Cohen, H. B. Injective envelopes of Banach spaces, 723.

Conner, P. E. and Floyd, E. E. Periodic maps which preserve a complex structure, 574. 
The SU-bordism theory, 670.

Curjel, C. R. See Arkowitz, M.

Curry, H. B. Combinatory recursive objects of all finite types, 814.

Curtis, C. W. Groups with a Bruhat decomposition, 357.

Dekker, J. C. E. The recursive equivalence type of a class of sets, 628.

Dreben, Burton and Aanderaa, Stål. Herbrand analyzing functions, 697.

Duda, Edwin. A locally compact separable metric space is almost invariant under a closed mapping, 285.

Earle, C. J. The Teichmiiller space of an arbitrary Fuchsian group, 699.

Ehrenpreis, Leon. See Bers, Lipman.

Faith, Carl. Noetherian simple rings, 730.

Feldman, E. A. The geometry of immersions. II, 600.

Figà-Talamanca, Alessandro. Multipliers of p-integrable functions, 666.

Finn, Robert. On normal metrics, and a theorem of Cohn-Vossen, 772.

Floyd, E. E. See Conner, P. E.

Fulkerson, D. R. and Gross, O. A. Incidence matrices with the consecutive 1's property, 681.

Gardner, L. T. On isomorphisms of $C^{*}$-algebras, 788.

Gerstenhaber, Murray. On the Galois theory of inseparable extensions, 561.

Goldstein, A. A. Convex programming in Hilbert space, 709.

Gottlieb, D. H. and Rothman, N. J. Contractibility of certain semigroups, 756.

Greathouse, Charles. Locally fat strings, 415.

The equivalence of the annulus conjecture and the slab conjecture, 716.

Green, P. S. A cohomology theory based upon self-conjugacies of complex vector bundles, 522.

Greenleaf, F. P. Norm decreasing homomorphisms of group algebras, 536.

Greiner, P. C. Eigenfunction expansions and scattering theory for perturbed elliptic partial differential operators, 517.

Gross, O. A. See Fulkerson, D. R.

Haimo, D. T. Variation diminishing transformations, 271.

Harary, Frank. See Beineke, L. W.

Harris, W. A., Jr. and Sibuya, Y. Note on linear difference equations, 123.

Hellerstein, S. and Korevaar, J. The real values of an entire function, 608.

Henriksen, Melvin and Isbell, J. R. Averages of continuous functions on countable spaces, $287,841$.

Hirsch, M. W. and Milnor, John. Some curious involutions of spheres, 372.

Hirschman, I. I., Jr. Finite section Wiener-Hopf equations on a compact group with ordered dual, 508.

See Baxter, Glen.

Hofmann, K. H. and Mostert, P. S. Irreducible semigroups, 621.

Totally ordered D-class decompositions, 765.

Hsiang, Wu-yi. On the unknottedness of the fixed point set of differentiable circle group actions on spheres-P. A. Smith conjecture, 678.

Hunt, R. A. An extension of the Marcinkiewicz interpolation theorem to Lorentz spaces, 803.

Hurd, A. E. and Schubert, C. F. An approach to quasilinear elliptic problems, 732.

Ionescu Tulcea, A. Ergodic properties of isometries in $L^{p}$ spaces, $1<p<\infty, 366$.

Ionescu Tulcea, A. and Ionescu Tulcea, C. On the lifting property. III, 193.

Ionescu Tulcea, C. See Ionescu Tulcea, A.

Isbell, J. R. Injective envelopes of Banach spaces are rigidly attached, 727. 
Isbell, J. R. See Henriksen, Melvin.

Itô, Takashi and Amemiya, Ichiro. A simple proof of the theorem of P. J. Cohen, 774 .

Kahane, J.-P. and Salem, R. Distribution modulo 1 and sets of uniqueness, 259.

Kantorovitz, Shmuel. Classification of operators by means of the operational calculus, 316.

Kaplan, Stanley. Differential equations in which the Poisson process plays a role, 264.

Kato, Tosio. Demicontinuity, hemicontinuity and monotonicity, 548.

Katznelson, Y. Sets of uniqueness for some classes of trigonometrical series, 722.

Keisler, H. J. On cardinalities of ultraproducts, 644 .

Kinney, J. R. and Pitcher, T. S. The dimension of the support of a random distribution function, 161 .

Klingenberg, Wilhelm. On the number of closed geodesics on a riemannian manifold, 279.

Kobayashi, Shoshichi and Nagano, Tadashi. A theorem on filtered Lie algebras and its applications, 401.

Kolchin, E. R. The notion of dimension in the theory of algebraic differential equations, 570.

Korevaar, J. See Hellerstein, S.

Krall, A. M. On the real parts of zeros of exponential polynomials, 291.

Kuroda, S. T. On a stationary approach to scattering problem, 556.

Lallement, Gérard and Petrich, Mario. Some results concerning completely 0-simple semigroups, 777.

Landau, H. J. A sparse regular sequence of exponentials closed on large sets, 566.

Lax, P. D. and Phillips, R. S. Scattering theory, 130.

Lazard, M. See Bass, H.

Levinson, N. and McKean, H. P., Jr. Weighted trigonometrical approximation on $R^{1}$ with application to the germ field of a stationary Gaussian process, 128.

Lumer, G. Analytic functions and Dirichlet problem, 98.

Lynch, R. E., Rice, J. R. and Thomas, D. H. Tensor product analysis of partial difference equations, 378.

McCrimmon, K. Jordan algebras of degree 1, 702.

McKean, H. P., Jr. See Levinson, N.

McMillan, D. R., Jr., Taming Cantor Sets in $E^{n}, 706$.

Mahowald, M. E. See Barratt, M. G.

Marcus, Marvin. On two classical results of $I$. Schur, 685.

Marcus, Marvin and Minc, Henryk. Inequalities for general matrix functions, 308.

Miller, R. K. On almost periodic differential equations, 792.

Milnor, John. See Hirsch, M. W.

Minc, Henryk. See Marcus, Marvin.

Mostert, P. S. See Hofmann, K. H.

Nagano, Tadashi. See Kobayashi, Shoshichi.

Neuwirth, Lee. In-groups and imbeddings of $n$-complexes in $(n+1)$-manifolds, 737.

Nijenhuis, Albert and Richardson, R. W., Jr. Cohomology and deformations of algebraic structures, 406.

Ono, Takashi. On the relative theory of Tamagawa numbers, 325 .

Palais, R. S. and Smale, S. A generalized Morse theory, 165.

Parr, J. T. Cohomology of cyclic groups of prime square order, 427.

Pearcy, Carl. See Brown, Arlen.

Petrich, Mario. See Lallement, Gérard.

Phillips, R. S. See Lax, P. D. 
Pitcher, T. S. See Kinney, J. R.

Pollard, Harry. A sharp form of the Virial theary, 708.

Pugh, C. C. Cross-sections of solution funnels, 580.

The closing lemma and structural stability, 584.

Quine, W. V. and Wang, Hao. On ordinals, 297.

Radlow, James. A two-dimensional singular integral equation of diffraction theory, 596.

Raimi, R. A. Minimal sets and ergodic measures in $\beta N-N, 711$.

Restrepo, Guillermo. Differentiable norms in Banach spaces, 413.

Rice, J. R. See Lynch, R. E.

Richardson, R. W. Jr. See Nijenhuis, Albert.

Robertson, Neil. The smallest graph of girth 5 and valency 4,824

Rockafellar, R. T. Duality theorems for convex functions, 189.

Rosen, R. H. The five dimensional polyhedral Schoenflies theorem, 511.

Rosenberg, Harold. A generalization of Morse-Smale inequalities, 422.

Rothman, N. J. See Gottlieb, D. H.

Rovnyak, James. See de Branges, Louis.

Rudin, Walter. Essential boundary points, 321.

Sakai, Shôichirô. On the reduction theory of von Neumann, 393.

Salem, R. See Kahane, J. P.

Sato, Daihachiro and Straus, E. G. Rate of growth of Hurwitz entire functions and integer valued entire functions, 303.

Sawyer, S. On inequalities of weak type, 637.

Schäffer, J. J. On Floquet's theorem in Hilbert spaces, 243.

Schanuel, S. On heights in number fields, 262.

Schoenberg, I. J. Spline interpolation and best quadrature formulae, 143.

Schubert, C. F. See Hurd, A. E.

Segal, I. E. Infinite-dimensional irreducible representations of compact semi-simple groups, 155.

Sell, G. R. A note on the fundamental theory of ordinary differential equations, 529.

Serre, J.-P. See Bass, H.

Shepp, L. A. Recurrent random walks with arbitrarily large steps, 540 .

Shih, Weishu. On the group $\mathcal{E}[X]$ of homotopy equivalence maps, 361 .

Sibuya, Y. See Harris, W. A., Jr.

Singletary, W. E. A complex of problems proposed by Post, 105.

Correction to "A complex of problems proposed by Post," 826.

Smale, S. See Palais, R. S.

Srinivasacharyulu, K. Deformations of riemannian structures, 178.

Stein, S. K. See Chakerian, G. D.

Steinberg, Arthur. See Baumslag, Gilbert.

Stout, E. L. Some theorems on bounded holomorphic functions, 419.

Straus, E. G. See Sato, Daihachiro.

Suzuki, Yoshindo. A complete classification of the $\Delta_{2}{ }^{1}$-functions, 246.

Tamura, T. Operations on binary relations and their applications, 113.

Thomas, D. H. See Lynch, R. E.

Tully, E. J., Jr., The equivalence, for varieties of semigroups, of two properties concerning congruence relations, 399.

Wall, C. T. C. An obstruction to finiteness of CW-complexes, 269.

Wang, Hao. See Quine, W. V.

Weinberger, H. F. On bounding harmonic functions by linear interpolation, 525 .

Werner, Helmut. On the local behavior of the rational Tschebyscheff operator, 554. 
Wijsman, R. A. Convergence of sequences of convex sets, cones and functions, 186.

Wilansky, Albert and Zeller, Karl. Erratum to " $A$ biorthogonal system which is not a Toeplitz basis," 327.

Wilcox, C. H. The domain of dependence inequality for symmetric hyperbolic systems, 149

Wilker, Peter. Double loops and ternary rings, 543.

$\mathrm{Wu}, \mathrm{H}$. Decomposition of riemannian manifolds, 610 .

Youngs, J. W. T. Irreducible graphs, 404.

Zachmanoglou, E. C. An example of slow decay of the solution of the initial-boundary value problem for the wave equation in unbounded regions, 633.

Zarantonello, E. H. The closure of the numerical range contains the spectrum, 781 .

Zeller, Karl. See Wilansky, Albert.

\section{RESEARCH PROBLEMS}

Brauer, George. Sets of divergence of exponential series, 661.

Dantzig, G. B. Eight unsolved problems from mathematical programming, 499.

Daykin, D. E. Space filling with unequal cubes, 340.

DeMarr, Ralph. Extension of commuting mappings, 500.

Golomb, S. W. Randon permutations, 747.

Horadam, E. M. Number theory or numerical semigroups, 339.

Isbell, J. R. Piecewise polynomial functions, 339.

Korfhage, R. R. On a sequence of prime numbers, 341.

Correction to "On a sequence of prime numbers," 747.

Parker, E. T. Finite combinatorial set theory, 499.

Redheffer, R. M. Operators on Hilbert space, 746.

Sato, Daihachiro. Function theory, 338.

\section{BOOK REVIEWS}

Abraham, Ralph. See Lang, Serge.

Auslander, L., Green, L. and Hahn, F. Flows on homogeneous spaces. W. H. Gottschalk, 649.

Auslander, Louis and Mackenzie, R. E. Introduction to differentiable manifolds. Robert Hermann, 331.

Auslander, Louis. See Helgason, S.

Barut, A. O. Electrodynamics and classical theory of fields and particles. Robert Hermann, 658.

Buchsbaum, D. A. See MacLane, Saunders.

Crowell, R. H. and Fox, R. H. Introduction to knot theory. L. P. Neuwirth, 235.

Chung, K. L. See Rényi, A.

Epstein, D. B. A. Cohomology operations. Lectures by N. E. Steenrod. R. H. Szczarba, 482.

Flanders, Harley. Differential forms with applications to the physical sciences. Robert Hermann, 483.

Fox, R. H. See Crowell, R. H.

Friedman, Avner. Generalized functions and partial differential equations. F. Treves, 94.

Gelfond, A. E. and Linnik, U. V. Elementary methods in analytic number theory. L. J. Mordell, 653.

Gottschalk, W. H. See Auslander, L., Green, L. and Hahn, F.

Helgason, S. Differential geometry and symmetric spaces. Louis Auslander, 227.

Hermann, Robert. See Auslander, Louis and Mackenzie, R. E. 
See Barut, A. O.,

See Flanders, Harley.

See Kobayashi, Shoshichi.

Hewitt, Edwin. See Hildebrandt, T. H.

Hildebrandt, T. H. Introduction to the theory of integration. Edwin Hewitt, 490.

Hilton, P. J. and Wylie, S. Homology theory. An introduction to algebraic topology. W. S. Massey, 333.

Kahane, J.-P. and Salem, Raphael. Ensembles parfaits et séries trigonométriques. Reviewed with Algebraic numbers and Fourier analysis (Salem). Walter Rudin, 487.

Kahane, J. P. See Rudin, Walter.

Kobayashi, Shoshichi and Nomizu, Katsumi. Foundations of differential geometry. Robert Hermann, 232.

Lang, Serge. Introduction to differentiable manifolds. Ralph Abraham, 225.

Diophantine geometry. L. J. Mordell, 491.

MacLane, Saunders. Homology. D. A. Buchsbaum, 329.

Massey, W. S. See Hilton, P. J. and Wylie, S.

Mordell, L. J. See Gelfond, A. E. and Linnik, U. V. See Lang, Serge.

Neuwirth, L. P. See Crowell, R. H.

Nomizu, Katsumi, See Kobayashi, Shoshichi.

Rényi, A. Wahrscheinlichkeitsrechnung mit einem Anhang über Informationstheorie. K. L. Chung, 224.

Rudin, Walter. Fourier analysis on groups, J.-P. Kahane, 230.

- See Kahane, J.-P.

- See Salem, Raphael.

Salem, Raphael. Algebraic numbers and Fourier analysis. Reviewed with Ensembles parfaits et sếries trigonométriques (Kahane and Salem). Walter Rudin, 487.

Szczarba, R. H. See Epstein, D. B. A.

Treves, F. See Friedman, Avner.

Volterra, Vito. Opere Mathematiche. Memorie e Note. A. Weinstein, 335.

Weinstein, A. See Volterra, Vito.

\section{REPORTS OF MEETINGS AND MISCELLANEOUS ARTICLES}

By-Laws of the American Mathematical Society, 833.

Council and Board of Trustees-1963, 198.

Endowment Fund and Special Funds of the Society, 829.

Curtis, M. L. Reports of Meetings of the American Mathematical Society: The November Meeting in Atlanta, 239.

Green, J. W. and Curtis, M. L. Reports of Meetings of the American Mathematical Society: The Annual Meeting in Miami, 343.

Green, J. W. and Pitcher, Everett. Reports of Meetings of the American Mathematical Society: The Summer Meeting in Amherst, 748.

Green, J. W. and Sherman, Seymour. Reports of Meetings of the American Mathematical Society: The April Meeting in Chicago, 503.

Pierce, R. S. Reports of Meetings of the American Mathematical Society: The November Meeting in Pasadena, 240; The April Meeting in Reno, 501; The June Meeting in Pullman, 665.

Pitcher, Everett. Reports of Meetings of the American Mathematical Society: The October Meeting in New York, 97; The February Meeting in New York, 356; The April Meeting in New York, 502. 
Report of the Treasurer, 662.

Shapiro, V. L. Fourier series in several variables, 48.

Youngs, J. W. T. Reports of Meetings of the American Mathematical Society: The November Meeting in Madison, 242.

\section{INVITED ADDRESSES}

de Branges, Louis. New and old problems for entire functions, 214.

242 .

Brown, Morton, 343.

Calabi, Eugenio, 754.

Carlitz, Leonard, 240.

Dantzig, G. B., 501.

Eggleston, H. G., 665.

Fejes T6th, L. What the bees know and what they do not know, 468. 503.

Guillemin, V. W. and Sternberg, Shlomo, An algebraic model of transitive differential geometry, 16.

Hall, Marshall, Jr., 501.

Helgason, Sigurdur. A duality in integral geometry; some generalizations of the Radon transform, 435.

Hironaki, Heisuke, 356.

Igusu, Jun-ichi, 754.

Kahane, J.P., 97.

Lacunary Taylor and Fourier series, 199.

Kohn, J. J., 502.

A priori estimates in several complex variables, 739.

Lehmer, D. H., 240.

Le Veque, W. J., 240.

Loewner, Charles. On semigroups in analysis and geometry, 1.

Morrey, C. B., 748.

Mumford, D. H., 754.

Nitsche, J. C. C., 503.

Rényi, A., 503.

Sard, Arthur, 748.

Shapiro, V. L. Harmonic analysis and the theory of cochains, 447.

Stallings, John, 748.

Sternberg, Shlomo. See Guillemin, V. W.

Whiteman, A. L., 240.

Wright, F. B., 239. 